\title{
Evaluation of topological planarity and reliability for interference reduction in radio sensor networks
}

\author{
Kamil Staniec ${ }^{*}$ and Grzegorz Debita
}

\begin{abstract}
One of the key factors in distributed sensor networks is their reliability in packets delivery even in the event of failure of one or a few nodes. Another factor-often underestimated-is the intra-network interference experienced by individual nodes from other transmitting counterparts. Common network spanning algorithms, however, neither posses the reliability feature nor keep the interference at minimum. It can be demonstrated that by enforcing a planar network topology combined with the requirement of minimum-pathloss between connections, one can achieve a gain in signal-to-noise and interference ratio (SNIR) by a few decibels with respect to standards spanning methods. The use of directional antennas and controlled duty cycle improves the situation even further-by carefully controlling the beamwidth, one may adjust the SNIR to a desired level at a high range of dynamics.
\end{abstract}

Keywords: sensor network, SNIR, ZigBee, directional antennas, duty cycle, radio interference

\section{Introduction}

A wireless sensor network (WSN) begins with an unorganized deployment of physical sensors connected to transmit/receive modules. Such a system of sensors can only be discussed in terms of a network if logical and physical connections have been established between these modules-a process called hence forth the network spanning. There are multiple spanning methods, of which two are particularly common, namely the ZigBee native method (or Minimum Spanning Tree, MST) and Stojmenovič algorithm (or Local MST algorithms, LMST), as described in [1-3]. The article provides a comparative analysis of these two methods with one which combines two features not encountered in the other algorithms, i.e., planarity and reliability (referred to from now on as a planar algorithm). The reliability should be an invaluable feature of sensor networks, particularly in situations where the lives of humans or living stock are dependent of it, e.g., networks for early fire or flood prevention. It is therefore crucial to ensure uninterrupted network operation even upon failure of some of the network elements scattered across a given

\footnotetext{
* Correspondence: kamil.staniec@pwr.wroc.pl

Institute of Telecommunications, Teleinformatics and Acoustics, Wroclaw University of Technology, Wroclaw, Poland
}

area. However, current popular network spanning methods are deficient as regards assurance of the network reliability (see [4-9]) and the topological planarity. The latter feature, particularly when combined with the use directional antenna, can greatly contribute to the minimization of the intra-network radio interference as will be demonstrated in the article.

The purpose of this article is therefore to present a quantitative analysis of the extent to which some improvements incorporated to the existing network spanning methods can enhance the distributed sensor network operation. These improvements are the planar network topology and reliability. All results presented here will refer to the performance of the ZigBee standard based on IEEE 802.15.4 specification [10] operating in the $2.4 \mathrm{GHz}$ ISM (Industrial, Scientific, Medical).

The organization of the article is as follows: Section 2 gives an introduction to standard network spanning methods (MST and LMST) and the reliable planar algorithm. Section 3 describes three basic parameters that will be investigated, i.e., signal-to-noise and interference ratio (SNIR), the antenna beamwidth, and duty cycle (DC). Section 4 serves as an introduction to the simulation environment used to generate results. Sections 5-7 provide results of simulations indicating how some 
operational mesh network parameters react to changes in the parameters mentioned in Section 3. All outcomes are presented in a manner allowing to compare the network performance in LMST, MST, and the reliable planar algorithm.

\section{Some remarks on the topological planarity and network reliability in WSN}

For the modeling of mesh systems the authors used theorems of the graph theory, one of which is the Gabriel's graph (GG). It is constructed by appropriately triangulating the network nodes: for every pair of interconnected nodes $u$ and $v$ separated by a distance $d$ (along the edge $l$ ) no other nodes can exist within the circular area of a diagonal $l$ located between $u$ and $v$ (more on the subject can be found in [1]). An example of GG is depicted in Figure 1a where the circular area stretched between nodes nos. 1 and 2 must be free from the presence of any other nodes (the same rule applies to all other pairs of connected nodes). Another important feature of GG is its intrinsic topological planarity which-as will be demonstrated in later sections-is very advantageous for radio links with directional antennas. Also, in order to draw GG, either the nodes' locations or the distances between them have to be known prior to making the graph of connections (links). As for the planarity feature, it is defined by Kuratowski theorem (see [7]) as follows: "a finite graph is planar if it does not contain a sub-graph which is an extended graph of graph $K_{5}$ (a full graph of five vertices) or graph $K_{3,3}$ (a dual full graph of six vertices in which three vertices are connected with each of the remaining three)" (see Figure 2).

An example of a network spanned with a classical form of GG is presented in Figure 1a. It is easy to notice that the structure does not satisfy the reliability theorem [6-8] which states (in a simplified version) that when each node has at least two neighbor nodes via which any other node in the network can be reached, such a network is regarded to be reliable. It is a vital feature to make the network survive single-node failures, whereas in Figure 1a the node no. 4 would be isolated from the rest of the network if node no. 1 failed. Clearly, this

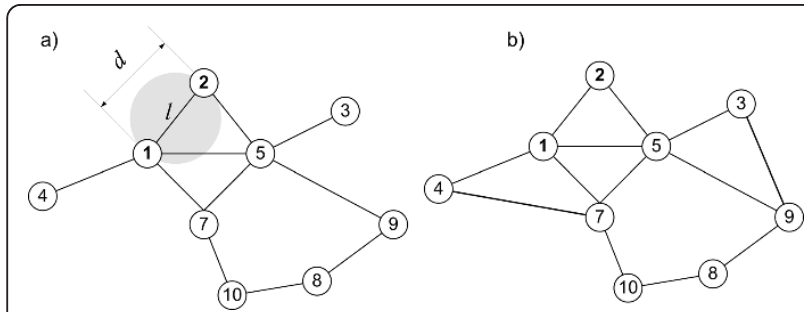

Figure 1 Examples of planar graphs organized with different spanning algorithms: (a) the original GG and (b) the modified (planar) GG.

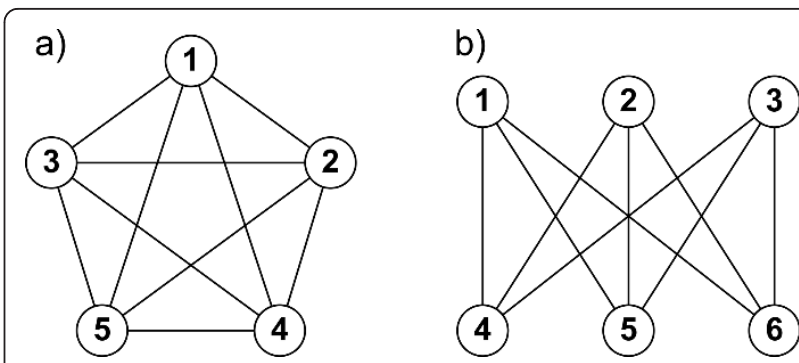

Figure 2 Auxiliary graphs in Kuratowski theorem: (a) $K_{5}$ and (b) $K_{3,3}$.

situation could be aided by creating an additional backup link (in this case from nodes 4 to 7 -see Figure 1b) and thus providing redundancy in the network. This is the first modification incorporated to GG by the authors (details given in [9]). The second one consisted in forcing the nodes interconnections to be set up only between the closest neighbor nodes (characterized by the smallest pathloss) rather than between those that first respond to the setup call (as is with ZigBee and Stojmenovič algorithms). This modification protects from establishing energy-costly links such as between nodes 17 and 20 in Figure 3b. Results of an example distributed sensor network consisting of 20 nodes and spanned with the ZigBee, Stojmenovič, and the reliable planar algorithm are shown for comparison in Figure 3.

Since it is assumed that nodes are located at some height over ground with no intervening obstacles between them, a simple (yet applicable to open-space areas) Two-Slope propagation model has been chosen (see [11] for details), given by Equation (1) where $d_{\text {brk }}$ is defined as $4 \pi h_{1} h_{2} / \lambda, h_{1}$ and $h_{2}$ denoting heights of the transmit and receive antennas, respectively, $\lambda$ the wavelength, $f_{\mathrm{MHz}}$ frequency in $\mathrm{MHz}$, and $d$ being a distance between both sensor antennas in $\mathrm{km}$.

$$
L_{\mathrm{dB}}= \begin{cases}32.45+20 \log f_{\mathrm{MHz}}+20 \log d_{\mathrm{km}} & d \leq d_{\mathrm{brk}} \\ 20 \log \left(h_{1} h_{2} / d_{\mathrm{km}}^{2}\right) & d>d_{\mathrm{brk}}\end{cases}
$$

In the following sections, it will be shown quantitatively that if the topological planarity condition is maintained during the mesh networks design stage (taking as
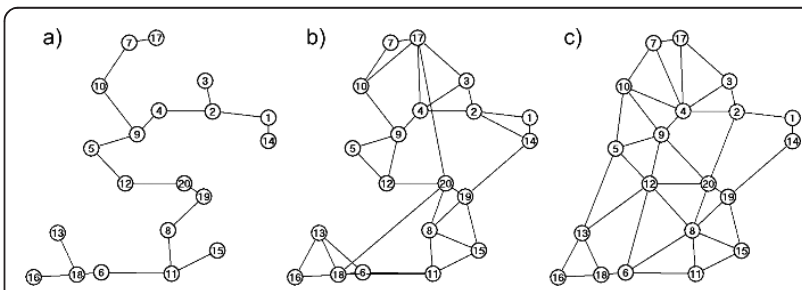

Figure 3 Examples of a distributed sensor network spanned with different algorithms: (a) ZigBee (b) Stojmenovič, and (c) the reliable planar algorithm. 
an example technology the IEEE 802.15.4, or ZigBee), a remarkably reduced intra-network radio interference level should be expected (apart from increased reliability) in comparison to standard LMST and MST methods.

\section{Items of interference analysis: SNIR, directional ARP, and DC}

Interference in WSN occurs mainly because even if direct line-of-sight directions between communicating nodes are not interfered, radio modules in the nodes can still hear unwanted transmissions from other nodes, due to (most usually) omni-directional antennas installed on them. The use of radiators with directional Antenna Radiation Patterns (ARP), as shown in Figure $4 \mathrm{a}$, could aid the situation by constraining emissions/ receptions in only specified directions thus making the desirable transmissions immune to foreign transmissions arriving from angles outside the beamwidth (marked as $\theta_{3 \mathrm{~dB}}$ in Figure 4a). An example is provided in Figure $4 \mathrm{~b}$ : the communication session between nodes 1 and 2 is only affected by interference from nodes 3 to 4 transmission but is deaf to radio transmission between node pairs 7-8 and 5-6 by attenuating those signals lying outside the main lobe. The topic of antenna directionality has been extensively addressed in literature (e.g., [12-19]) mostly from the point of view of the medium access control or energy-optimal routing strategies. In this article, in turn, it will be discussed in terms of its impact on the physical layer of the WSN, namely how it alleviates the intra-network radio interference. Lastly, it should be remembered that the application of directional antennas requires some sort of adaptive antenna system to be installed on board each transmitting node. Every time a new packet is to be sent, a neighbor node will randomly be selected out of the predefined set of least-pathloss nodes and the main lobe of transmitting antenna will switch its direction in order to concentrate its energy towards the selected neighbor (refer for more information on this issue to [20]).

The major metric for the intra-network radio interference is the SNIR-a basic parameter for performance evaluation in digital systems (in ZigBee operating at 2.4 $\mathrm{GHz}$ SNIR equals $+5 \mathrm{~dB}$ ). For the $k$ th node, it is given by Equation (2), where $S$ is the desired signal power in $\mathrm{W}, k_{\mathrm{B}}$ is the Boltzman's constant in $\mathrm{J} / \mathrm{K}, T$ is the ambient temperature in $\mathrm{K}$, BW channel bandwidth in $\mathrm{Hz}$, NF is the noise factor [-], $N$ is the total number of network nodes, whereas the last term in the denominator denotes the sum of radiations from all other nodes $\left(I_{n}\right)$ in the network (excluding, of course, the radiation from the $k$ th node for which SNIR is being calculated and the $l$ th node to which the node $k$ is transmitting, i.e., $I_{k}$ and $I_{l}$, respectively).

$$
\mathrm{SNIR}_{k \mid[\mathrm{dB}]}=10 \log \left(\frac{S}{N+\sum_{M / k, l]} I_{m}}\right)=10 \log \left(\frac{S}{k_{B} \cdot T \cdot \mathrm{BW} \cdot \mathrm{NF}+\sum_{\mathrm{M} /[k, I]} I_{m}}\right)
$$

The last aspect subject to investigations is the $D C$ expressed in percentage and understood in the following manner: a notation $D C=p \%$ is equivalent to the restraint that during the time period $T$ a given device will transmit only for $p \%$ of $T$ duration. Such a bound on $D C$ has been officially imposed on ZigBee devices operating at $868 \mathrm{MHz}(\mathrm{DC}<1 \%)$ [10] but none such limitation exists for the other two frequency bands. There is also a broad publication coverage on this aspect although mainly from energy-saving viewpoint (as in [21-24]), whereas in this article attention is laid on its effect on the SNIR improvement in the network.
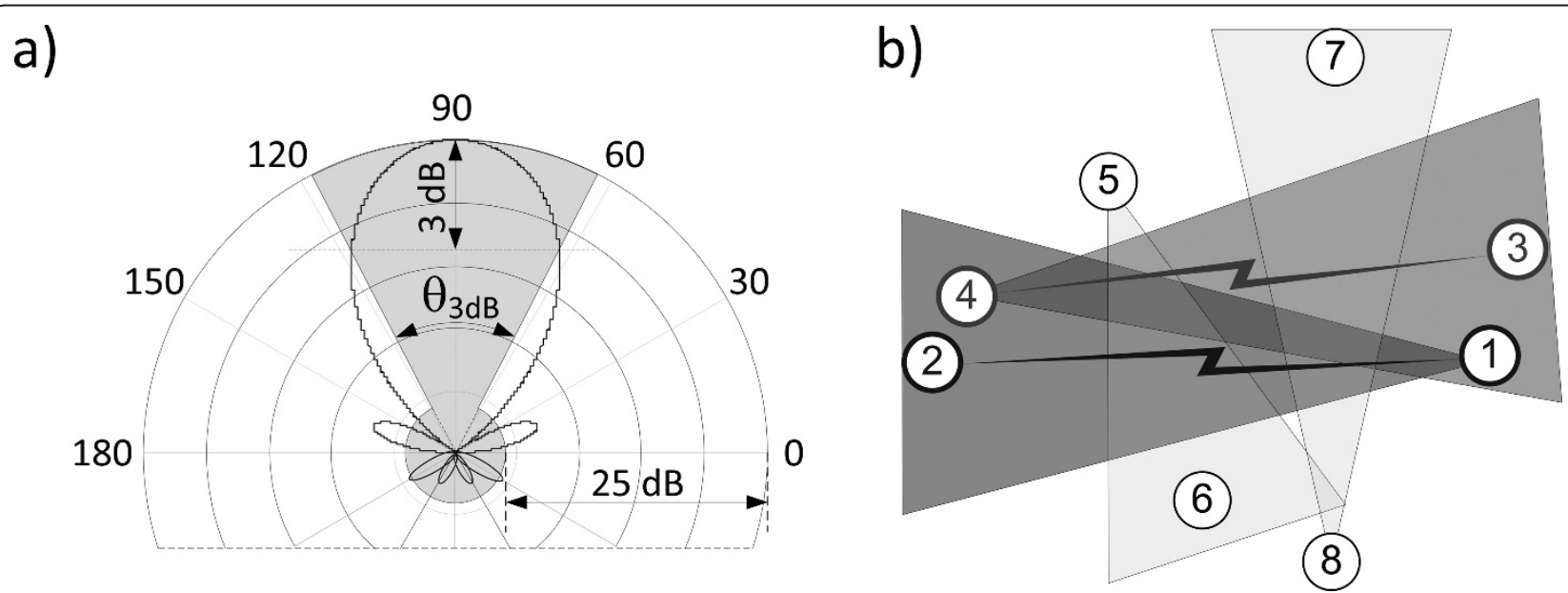

Figure 4 The idea and application of directional antennas: (a) a simplified model of the antenna ARP and (b) an example of interference reduction due to the use of directional antennas. 
In [4], it was demonstrated how both directional ARP and limited $D C$ can lead to reduction of intra-network interference and thus improve the network overall performance whereas in the investigations to follow the aim was to verify the extent to which the interference can be further reduced in the WSN if the planar topology is enforced by the spanning method in the network formation stage.

\section{Notes on simulations}

For the purpose of comparison between the ZigBee, Stojmenovič, and the planar network spanning algorithm, a software simulator has been created comprising two main components:

Matlab scripts to perform the network spanning algorithms on the random scenarios generated by the $\mathrm{C}++$ application (the component described below);

a C++ Builder application for (a) generating random node distribution scenarios for each population of nodes $M$ (i.e., from 10, 20 up to 100). These serve as input files to the Matlab scripts and (b) calculating SNIR based on the output files generated by the Matlab scripts.

In order to study the behavior of the ZigBee network, multiple scenarios were generated with a variable number of sensor nodes $(M=10-100)$, each located at $1.5 \mathrm{~m}$ above the ground and transmitting with the power 0 $\mathrm{dBm}$ and $0 \mathrm{dBi}$ gain. The nodes were Monte-Carlo distributed over a square area of $500 \times 500 \mathrm{~m}^{2}$.

\section{Analysis of results: SNIR dependency on the antenna beamwidth}

In the first step, the SNIR dependency on the antenna beamwidth $\theta_{3 \mathrm{~dB}}$ was investigated. Simulations were performed for multiple scenarios to determine the influence of the antenna beamwidth on the level of interference experienced by a statistical network node. Next, SNIR was found for every individual node and averaged over the whole network and later-over all scenarios generated for the given $M$ (Figure 5). Such an averaging procedure was intended to provide statistical robustness to results since values of SNIR obtained for individual network members were prone to vary considerably. As can be seen, the signal to interference obtained in networks forming planar topologies (solid lines) outperforms that achieved in networks organized with standard methods (namely ZigBee and Stojmenovič) by ca. 4-5 dB for the entire range of the antenna beamwidth $\theta_{3 \mathrm{~dB}}$. In other words, the planar topology allows wider beamwidth to sustain the same SNIR, which indicates that the planar network is intrinsically more resilient to the intra-network interference. This behavior is attributed to the fact that connections do not intersect each other therefore the number of interference sources lying within a node's 3 - $\mathrm{dB}$ angle $\left(\theta_{3 \mathrm{~dB}}\right)$ is lower.

Lastly, it is noteworthy that even for a "needle" ARP (i. e., with $\theta_{3 \mathrm{~dB}}=0^{\circ}$ ) the average SNIR is still a few decibels higher in the planar network than in the other networks. This is due to the feature implemented by the authors in the planar algorithm (recall Section 2) which allows links to be established only between nodes with the lowest pathloss (i.e., with the highest signal). Hence, even in the absence of any interference due to infinitely thin ARP (only the noise term remains in the denominator of Equation 2), the average desired signal $S$ will be expectedly higher in planar networks.

Both these features (i.e., the planarity and the minimum-pathloss neighbor selection) combined in the planar spanning algorithm, make the network operate at an energetic optimum as opposed to the two other methods where candidate nodes are selected on the "first-torespond" basis where it is not guaranteed that "the first" will be equivalent to "the closest" at all times (and indeed is not, as stems from simulations and measurements). An immediate advantage to be taken from the least-pathloss approach is an extended sensor network longevity since less energy is now needed to transfer data to the closest neighbor instead of a remote one.

One should note that the ZigBee devices will function properly if the minimum $\mathrm{SNIR}_{\min }$ of $5 \mathrm{~dB}$ is maintained, as given in [10] (represented by a dashed horizontal line in Figure 5). The value of the beamwidth for which a given curve crosses $\mathrm{SNIR}_{\min }$, therefore, establishes the maximum antenna $3-\mathrm{dB}$ angle $\theta_{3 \mathrm{~dB} \mid \mathrm{MAX}}$ to keep interference low compared to the desired signal. In order to cover the entire range of simulated nodes populations $(10,20, \ldots, 100)$, curves representing $\theta_{3 \mathrm{~dB} \mid \mathrm{MAX}}$ as a function of the number o nodes $M$ have been plotted in Figure 6a. It can be noticed that for sparsely deployed nodes $(M=10)$ the planarity allows to attain a statistically correct network functioning for antenna beamwidths wider by ca. $50^{\circ}$ compared to ZigBee and Stojmenovič algorithms (Figure $6 \mathrm{~b}$ ). Then, with a growing number of nodes, the best-fit trends in $\theta_{3 \mathrm{~dB} \mid \mathrm{MAX}}$ decline exponentially at a rate of $\exp (-0.2)$ regardless of the spanning method, to eventually converge to ca. $15^{\circ}$ of advantage of the planar spanning algorithm over the ZigBee, Stojmenovič counterparts, for mostly populated networks.

\section{Analysis of results: number of intereferors dependency on the antenna beamwidth}

It can be noticed from the definition of planar GG provided in Section 2 that the condition prohibiting the presence of nodes within a circle formed around any edge $l$ (Figure 1a) implies that the angles between successive connections will (on average) also tend to be 

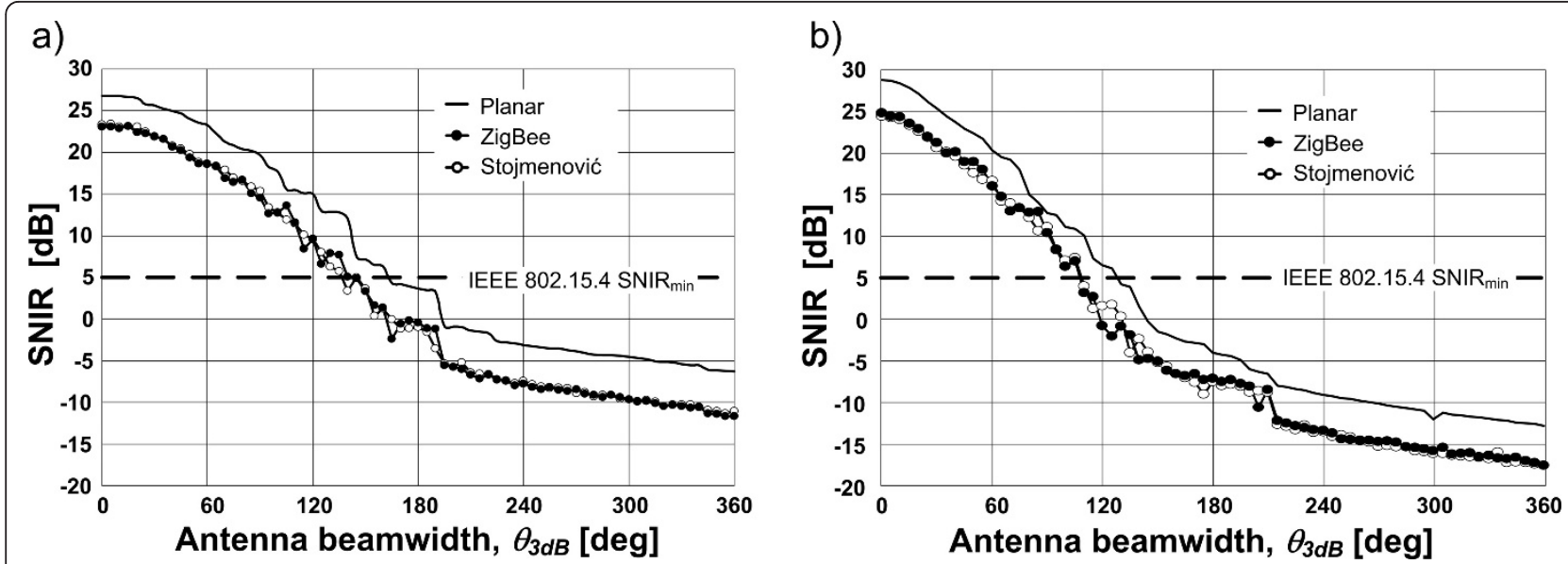

Figure 5 A dependency of SNIR as a function of $\boldsymbol{\theta}_{\mathbf{3 d B}}$ for a different number of sinks $\boldsymbol{M}$ : (a) $\boldsymbol{M}=20$ nodes and (b) $\boldsymbol{M}=90$ nodes.

wider than in networks without this constraint. This, in turn, will affect the number of interferers $(\Sigma I)$ "seen" by a statistical node within the radio visibility range (and subtended within $\theta_{3 \mathrm{~dB}}$ angle).

As can be noticed in Figure 7a, in planar networks $\Sigma I$ is apparently lower (and almost linear) than in ZigBeeor Stojmenovič-spanned networks. Quite interestingly this difference becomes most distinguished as the antenna beamwidth approaches $180^{\circ}$-the effect simply obtained by mounting a reflective screen on one side of the antenna. It is also a mid-point between two extremes where planarity causes no advantage (regarding only the number of interferers) over non-planar solutions, namely for $\theta_{3 \mathrm{~dB}}=0^{\circ}$ (a "needle" ARP) and $\theta_{3 \mathrm{~dB}}=360^{\circ}$ (omnidirectional ARP). The plots of differences $\Delta_{\Sigma I}$ between the number of interferers observed by a statistical node in networks organized with ZigBee $\left(\Sigma I_{\mathrm{ZigBee}}\right)$ and planar $\left(\Sigma I_{\text {planar }}\right)$ algorithms are shown in Figure $7 \mathrm{~b}$. Stojmenovič case has been omitted since its performance in this test is indistinguishably similar to that of ZigBee, as seen in Figure 7a. From the analysis of $\Delta_{\Sigma I}$ obtained for all investigated values of $M(10-100)$ it stems that in planar topologies a statistical node will be subject to disturbances from $9 \%$ less interferers than in non-planar ones.

\section{Analysis of results: DC dependency on the antenna beamwidth}

A DC, as described in Section 3, can be considered as a statistical means of reducing the intra-network interference by implementing a mechanism allowing transmission opportunities with some probability $D C$. The subject was given an in-depth attention in [4] where it was demonstrated that even under the most transmission-intensive circumstances (such as a sudden appearance of fire or flood in a given area which trigger multiple sensors simultaneously) if the transmit opportunity is restricted, there is a greater chance that a packet will pass through the network-otherwise it could be drowned out by numerous parallel transmissions

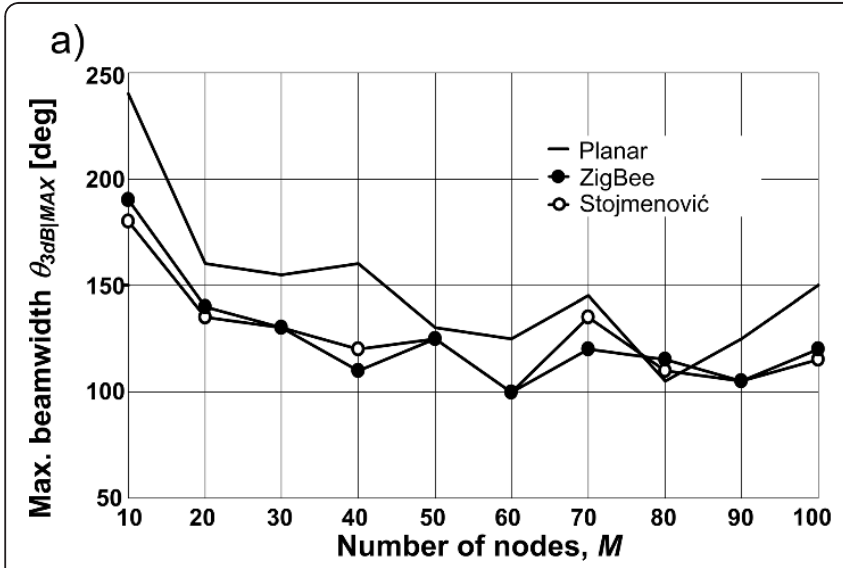

b)

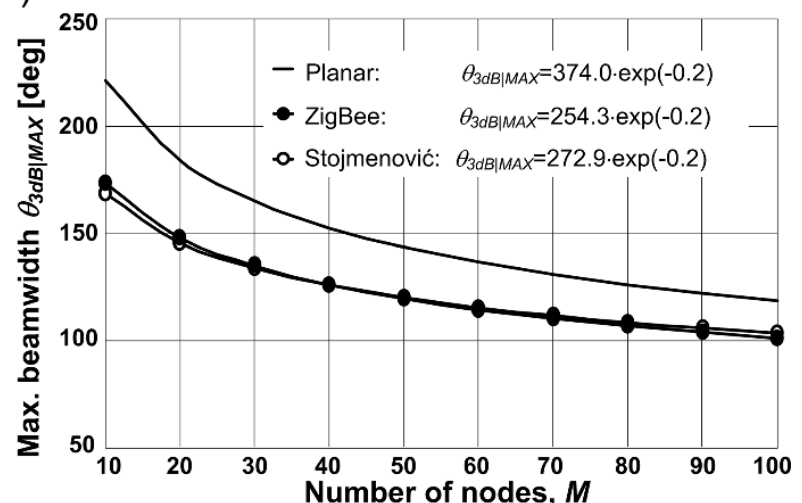

Figure 6 The maximum beamwidth dependency on the number of network nodes: (a) simulated; (b) exponential best-fit. 

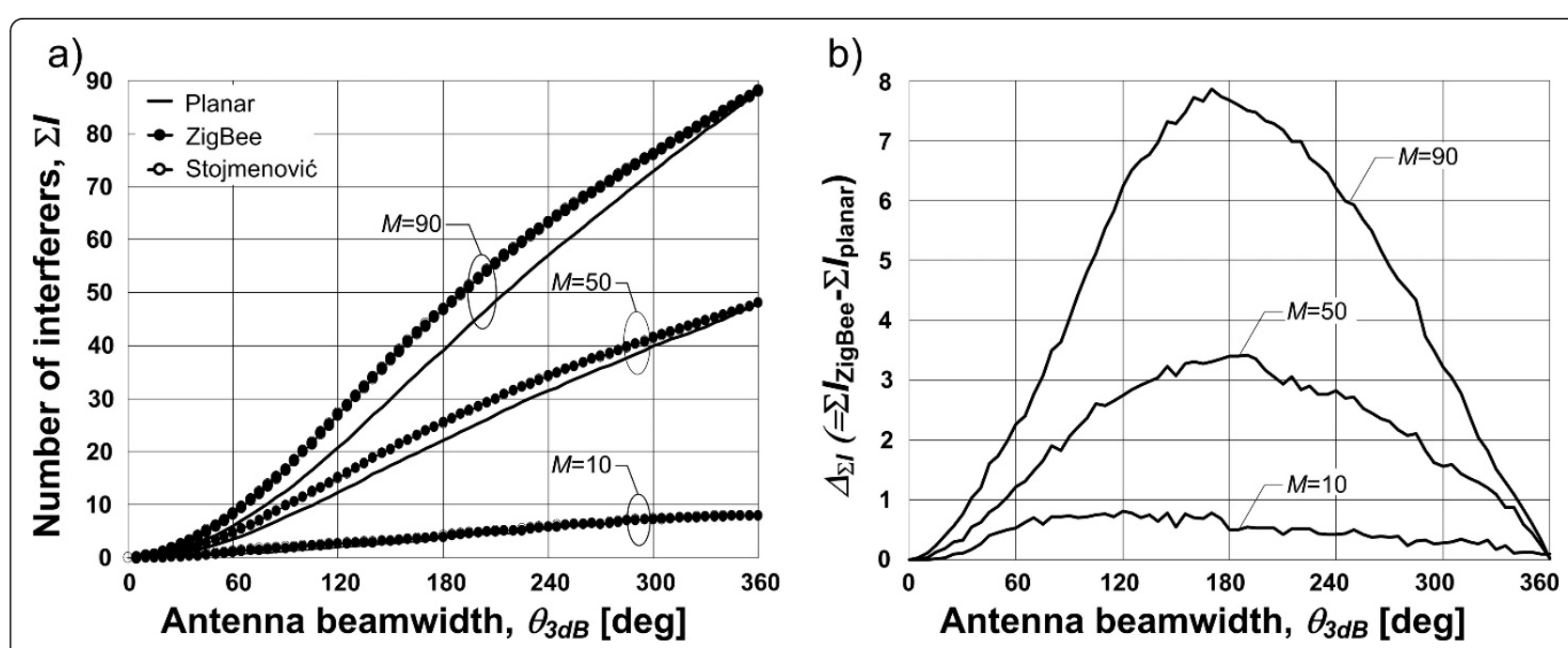

Figure 7 The effect of using directional antennas and planar topologies on different interference aspects: (a) the average number of interferers $\Sigma$; (b) the difference $\Delta_{\Sigma l}$ between $\Sigma$ l observed by a statistical node in an MST-spanned and a planar mesh network.

(carrying the same data) from other nearby sensor modules. In the current investigations, similarly as in Section 6 , only ZigBee algorithm plots will be presented, due to their high similarity with the Stojmenovič method. As shown in Figure 8a, the expected gain in SNIR in the planar method is on average higher by $4.15 \mathrm{~dB}$ than in the ZigBee algorithm and this offset appears to be maintained over the whole range of the antenna bandwidth and for all studied values of $D C$ (i.e., 1, 5, 10, 20, 30 up to $100 \%)$. As concerns the maximum antenna beamwidth $\theta_{3 \mathrm{~dB} / \text { MAX }}$ necessary to sustain SNIR at or above 5 $\mathrm{dB}$, results (Figure $8 \mathrm{~b}$ ) indicate an exponential relation with the $\mathrm{DC}$ (valid for $\mathrm{DC} \geq 30 \%$ ). $\theta_{3 \mathrm{~dB} / \mathrm{MAX}}$ in the reliable planar topology is allowed to be on average $34^{\circ}$ wider than in ZigBee algorithm to achieve the desirable SNIR.

\section{Summary and conclusions}

It has been demonstrated that two most popular network spanning methods, namely Stojmenovič and ZigBee algorithms, possess some significant drawbacks, namely they do not assure reliability and allow for energetically non-optimal transmissions to distant neighbors. Moreover, the latter feature-as was also shown in simulations-gives rise to excessive interference in the radio network. The authors have posed a thesis that the planar GG, after little modifications for reliability and a least-pathloss neighbor selection, should be an optimal algorithm of choice for forming mesh ad hoc networks. The justification of this assertion is provided throughout Sections 5-7 by investigating SNIR as a function of the transmit/receive antenna directivity. It was demonstrated (Section 5) that SNIR in reliable planar networks is

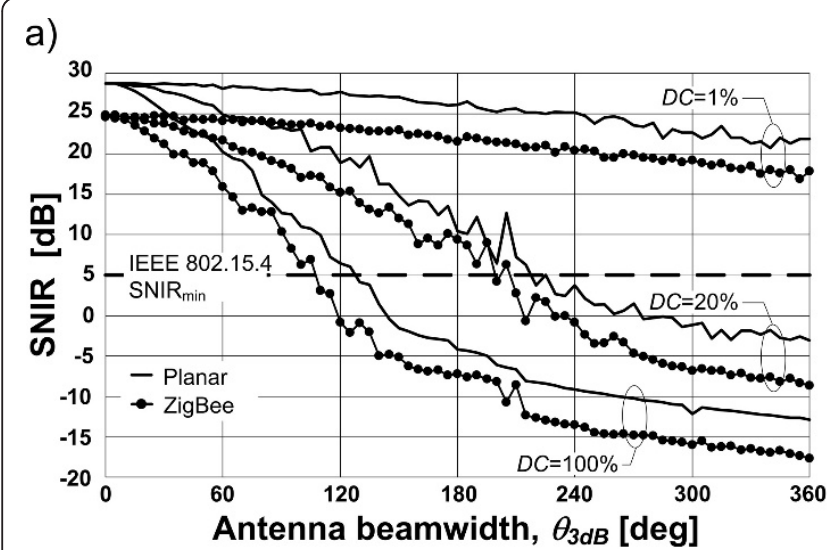

b)

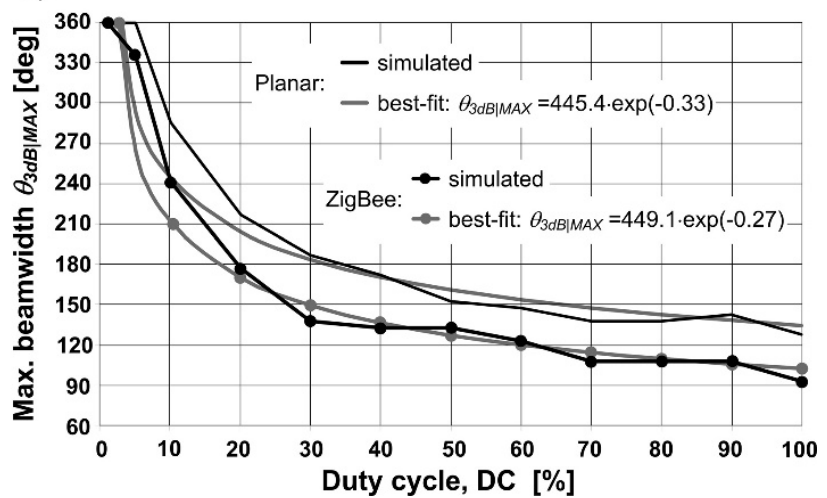

Figure 8 The effect of using directional antennas and planar topologies on different interference aspects: (a) the dependency of SNIR on $\mathrm{DC}$ and $\theta_{3 \mathrm{~dB}} ;$ (b) the maximum antenna beamwidth dependency on DC. 
improved by $4-5 \mathrm{~dB}$, at the beamwidth wider by up to $50^{\circ}$ than in ZigBee or Stojmenovič cases. An explanation of this effect is given in Section 6 where it is demonstrated that the average number of interfering nodes affecting a statistical node is lower by $9 \%$ in reliable planar networks than in Stojmenovič- and ZigBee-spanned networks. Lastly, it was shown in Section 7 that when a $\mathrm{DC}$ is imposed on network devices, an additional gain of $4.15 \mathrm{~dB}$ in SNIR is obtained for the reliable planar topology over the non-planar topologies.

\section{Acknowledgements}

This paper has been written partially as a result of realization of the project entitled: "Detectors and sensors for measuring factors hazardous to environment - modeling and monitoring of threats". The project financed by the European Union via the European Regional Development Fund and the Polish state budget, within the framework of the Operational Programme Innovative Economy $2007 \div 2013$. The contract for refinancing No. POIG.01.03.01-02-002/08-00.

This paper has been written partially as a result of realization of the project entitled: "Research on topology, distribution of sinks and packets routing with respect to reliability and energetic optimization in wireless sensor networks". The contract for refinancing No. B10089.

\section{Authors' contributions}

The work presented here was carried out in collaboration between both authors, all authors read and approved the manuscript. KS was responsible for the introductory part as a main contributor to Section 1. As the only author of Sections 3-8, he performed all interference investigations (together with the processing and analysis of results) which included: a) radio wave propagation aspects and b) C++ scripts for generating scenarios and computing SNIR vs. antenna beamwidth, duty cycle and the number of interferers.

GD, as a main contributor to Section 2 and a minor contributor to Section 1, created Matlab scripts for spanning network nodes with the reliable planar algorithm, the MST and LMST algorithms and performed necessary networkspanning calculations for scenarios generated by KS.

\section{Competing interests}

The authors declare that they have no competing interests.

Received: 30 October 2011 Accepted: 22 February 2012

Published: 22 February 2012

\section{References}

1. I Stojmenovič, Handbook of Sensor Networks-Algorithms and Architectures, John Wiley and Sons, Ottawa, (2004)

2. ML Shooman, Reliability of Computer Systems and Networks-Fault Tolerance, Analisys and Design, John Wiley \& Sons, (2002)

3. M Pióro, D Medhi, Routing, Flow, and Capacity Design in Communication and Computer Networks, (Morgan Kaufmann Publishers, San Francisco, 2004)

4. K Staniec, G Debita, Interference mitigation in WSN by means of directional antennas and duty cycle control. Wirel. Commun. Mobile Comput. 11 (2011). doi:10.1002/wcm.1089

5. G Debita, K Staniec, Reliable mesh network planning with minimization of intra-system interference, in Proceedings of Broadband Communication Information Technology \& Biomedical Applications BROADBAND-COM'09, Wrocław, Poland, 243-244 (15-July 2009)

6. H Frank, I Frisch, Analysis and design of survivable networks. IEEE Trans Commun Technol. 18(5), 501-518 (1970). doi:10.1109/TCOM.1970.1090419

7. D Narsingh, Graph Theory with Applications to Engineering and Computer Science, (Prentice-Hall, Inc, 1974)

8. LR Ford, DR Fulkerson, Maximal flow through a network. Can J Math. 8 399-404 (1956). doi:10.4153/CJM-1956-045-5

9. G Debita, Methods for optimal planning of mesh networks with consideration of reliability criteria, Doctoral dissertation, (Wroclaw University of Technology, Poland, 2010)
10. IEEE Std 802.15.4 ${ }^{\mathrm{TM}}$-2006 Part 15.4, Wireless Medium Access Control (MAC) and Physical layer (PHY) Specification for Low-Rate Wireless Personal Area Networks (WPANs)

11. SR Saunders, Antennas and Propagation for Wireless Communications Systems, 2nd edn. (John Wiley \& Sons, Ltd., Chichester, 2007)

12. H Jasani, $K$ Yen, Performance improvement using directional antennas in ad hoc networks. Int J Comput Sci Netw Secur. 6(6), 180-188 (2006)

13. RR Choudhury, NH Vaidya, Impact of directional antennas on ad hoc routing, in Proceedings of IFIP Personal and Wireless Communications (PWC) 2003, Venice, Italy, 590-600 (23-25 September 2003)

14. R Ramanathan, J Redi, C Santivanez, D Wiggins, S Polit, Ad hoc networking with directional antennas: a complete system solution. IEEE J Sel Areas Commun. 23(3), 496-506 (2005)

15. T ElBatt, T Anderson, B Ryu, Performance evaluation of multiple access protocols for ad hoc networks using directional antennas, Proceedings of IEEE Wireless Communications and Networking Conference (WCNC), New Orleans, Louisiana, USA, 2, 982-987 (16-20 March 2003)

16. A Munari, F Rossetto, M Zorzi, Cooperative cross layer MAC protocols for directional antenna ad hoc networks. ACM Mobile Comput Commun Rev. 12(2), 12-30 (2008). doi:10.1145/1394555.1394558

17. C Tran-Xuan, I Koo, An RSS-based localization scheme using direction calibration and reliability factor information for wireless sensor networks. KSII Trans Internet Inf Syst. 4(1), 45-61 (2010)

18. HN Dai, Throughput and delay in wireless sensor networks using directional antennas, in Proc 5th International Conference on Intelligent Sensors, Sensor Networks and Information Processing (ISSNIP), pp. 421-426 (7-10 December 2009)

19. Y Bashir, BO Jalel, Towards a classification of energy aware MAC protocols for wireless sensor networks Wirel Commun. Mobile Comput. 9(12), 1572-1607 (2009)

20. D Jenn, Y Loke, TCH Matthew, YE Choon, OC Siang, YS Yam, Distributed phased arrays and wireless beamforming networks. Int J Distrib Sensor Netw. 5(4), 283-302 (2009). doi:10.1080/15501320701863635

21. R Jurdak, CV Lopes, PA Baldi, A survey, classification and comparative analysis of medium access control protocols for ad hoc networks. IEEE Commun Surv Tutor. 6(1), 2-16 (2004)

22. Y Wei, J Heidemann, D Estrin, Medium access control with coordinated adaptive sleeping for wireless sensor networks. IEEE/ACM Trans Netw. 12(3), 493-506 (2004). doi:10.1109/TNET.2004.828953

23. MJ Miller, NH Vaidya, A MAC protocol to reduce sensor network energy consumption using a wakeup radio. IEEE Trans Mobile Comput. 4(3), 228-242 (2005)

24. AA Moshaddique, SM Riazul Islam, K Kwak, Energy saving mechanisms for MAC protocols in wireless sensor networks. Int J Distrib Sensor Netw. 2010, Article ID 163413, 16 (2010)

doi:10.1186/1687-1499-2012-56

Cite this article as: Staniec and Debita: Evaluation of topological planarity and reliability for interference reduction in radio sensor networks. EURASIP Journal on Wireless Communications

and Networking 2012 2012:56.

\section{Submit your manuscript to a SpringerOpen ${ }^{\mathcal{O}}$ journal and benefit from:}

- Convenient online submission

- Rigorous peer review

- Immediate publication on acceptance

- Open access: articles freely available online

- High visibility within the field

- Retaining the copyright to your article

Submit your next manuscript at $>$ springeropen.com 